\title{
Spin-pump-induced spin transport in a thermally evaporated pentacene film
}

\section{Yasuo Tani, Yoshio Teki, and Eiji Shikoh}

\begin{tabular}{|c|l|}
\hline Citation & Applied Physics Letters, 107(24):202406 \\
\hline Issue Date & $2015-12$ \\
\hline Type & Journal Article \\
\hline Textversion & publisher \\
\hline Right & $\begin{array}{l}\text { This article may be downloaded for personal use only. Any other use requires prior } \\
\text { permission of the author and AIP Publishing. } \\
\text { The following article appeared in Applied Physics Letters 107, 242406 (2015) and } \\
\text { may be found at http://doi.org/10.1063/1.4938132. }\end{array}$ \\
\hline DOI & \begin{tabular}{l}
$10.1063 / 1.4938132$ \\
\hline
\end{tabular} \\
\hline
\end{tabular}

Self-Archiving by Author(s)

Placed on: Osaka City University Repository 


\section{Spin-pump-induced spin transport in a thermally evaporated pentacene film}

Yasuo Tani, Yoshio Teki, and Eiji Shikoh

Citation: Appl. Phys. Lett. 107, 242406 (2015); doi: 10.1063/1.4938132

View online: http://dx.doi.org/10.1063/1.4938132

View Table of Contents: http://aip.scitation.org/toc/apl/107/24

Published by the American Institute of Physics

\section{Articles you may be interested in}

Spin current relaxation time in thermally evaporated pentacene films

Applied Physics Letters 110, 032403 (2017); 10.1063/1.4974294

Conversion of spin current into charge current at room temperature: Inverse spin-Hall effect

Applied Physics Letters 88, 182509 (2006); 10.1063/1.2199473

Important role of magnetization precession angle measurement in inverse spin Hall effect induced by spin pumping

Applied Physics Letters 110, 022404 (2017); 10.1063/1.4973704

Planar-type spin valves based on low-molecular-weight organic materials with $\mathrm{La}_{0.67} \mathrm{Sr}_{0.33} \mathrm{MnO}_{3}$ electrodes Applied Physics Letters 92, 153304 (2008); 10.1063/1.2905288

Observation of spin-charge conversion in chemical-vapor-deposition-grown single-layer graphene Applied Physics Letters 105, 162410 (2014); 10.1063/1.4893574

Control of the spin to charge conversion using the inverse Rashba-Edelstein effect Applied Physics Letters 106, 172403 (2015); 10.1063/1.4919129

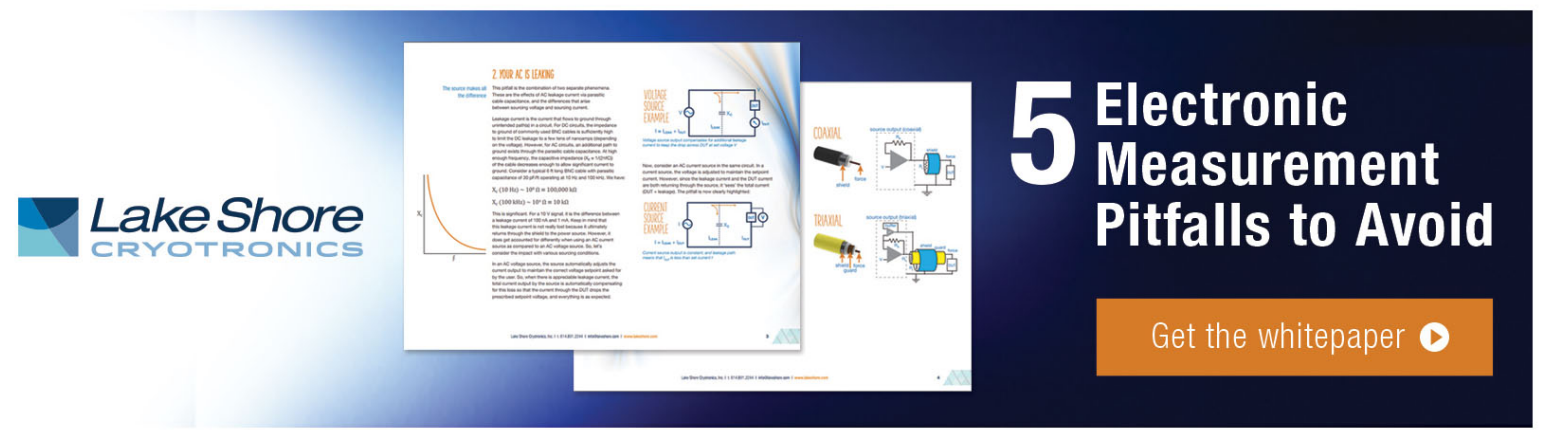




\title{
Spin-pump-induced spin transport in a thermally evaporated pentacene film
}

\author{
Yasuo Tani, ${ }^{1}$ Yoshio Teki, ${ }^{2}$ and Eiji Shikoh ${ }^{1, a)}$ \\ ${ }^{1}$ Graduate School of Engineering, Osaka City University, 3-3-138 Sugimoto, Sumiyoshi-ku, Osaka 558-8585, \\ Japan \\ ${ }^{2}$ Graduate School of Science, Osaka City University, 3-3-138 Sugimoto, Sumiyoshi-ku, Osaka 558-8585, Japan
}

(Received 11 September 2015; accepted 6 December 2015; published online 16 December 2015)

\begin{abstract}
We report the spin-pump-induced spin transport properties of a pentacene film prepared by thermal evaporation. In a palladium(Pd)/pentacene $/ \mathrm{Ni}_{80} \mathrm{Fe}_{20}$ tri-layer sample, a pure spin-current is generated in the pentacene layer by the spin-pumping of $\mathrm{Ni}_{80} \mathrm{Fe}_{20}$, which is independent of the conductance mismatch problem in spin injection. The spin current is absorbed into the Pd layer, converted into a charge current with the inverse spin-Hall effect in Pd, and detected as an electromotive force. This is clear evidence for the pure spin current at room temperature in pentacene films prepared by thermal evaporation. @ 2015 AIP Publishing LLC. [http://dx.doi.org/10.1063/1.4938132]
\end{abstract}

Pure spin current is dissipation-less information propagation in electronic devices. Carbon-based molecules are promising materials from the viewpoint of such spin transport because their spin-orbit interaction functioning as spin scattering centers is generally weak. In the field of molecular spintronics, ${ }^{1-10}$ pure spin current has been observed in graphene $^{2,3}$ and organic polymers, ${ }^{4,10}$ although the samples in those two cases were fabricated by the Scotch tape method and spin-coating, respectively, which are unsuitable for use in device fabrications.

In this study, we focus on a pentacene molecular film prepared by thermal evaporation (hereafter, "evaporatedpentacene" film) as a candidate material for spin transport. The reasons are as follows: First, vacuum thermal evaporation is better suited to preparing molecular films for electronic devices than spin-coating or the transfer of organic molecules. Second, pentacene has good crystallinity, even in the films formed by thermal evaporation, and shows relatively high electrical conductivity without any dopants. ${ }^{11}$ Pentacene, which is a $p$-type semiconductor, is well known as an active-layer material in organic field-effect transistors (OFETs). ${ }^{11}$ The carrier mobility for pentacene film is above $1 \mathrm{~cm}^{2} / \mathrm{V} \mathrm{s}$, which is the highest value for any OFET with molecular films fabricated by thermal evaporation and high enough for simple electronic devices. ${ }^{11}$ This means that numerous active carriers are present in the pentacene film (in this case, holes) to propagate the spin angular momentum. Moreover, pentacene shows photoconductivity for visible light, ${ }^{12}$ where the spin transport properties of pentacene can be controlled through light irradiation. This is an advantage for the use of molecular materials in future applications of spintronics over the use of inorganic materials. To date, spin transport in pentacene films has been studied using a spin-polarized charge current, as distinct from pure spin current, has been studied, ${ }^{5,6}$ although there is a conductance mismatch problem ${ }^{13,14}$ between the ferromagnetic electrode as a spin injector and pentacene. This problem lowers the spin injection efficiency and produces errors in the estimation of spin transport properties. In the present study, we show clear evidence for spin transport in a pentacene film at room

${ }^{\text {a)} E-m a i l: ~ s h i k o h @ e l e c . e n g . o s a k a-c u . a c . j p ~}$ temperature (RT) by using a pure spin current induced by spinpumping. ${ }^{15,16}$ In this case, the conductance mismatch problem related to spin injection with spin-pumping is negligible. ${ }^{17-19}$

Our sample structure and experimental set up is illustrated in Figure 1. Spin transport in pentacene is observed as follows: in palladium(Pd)/evaporated-pentacene/ $\mathrm{Ni}_{80} \mathrm{Fe}_{20}$ tri-layer samples, a spin-pump-induced pure spin current, $J_{\mathrm{S}}$, driven by ferromagnetic resonance (FMR) $)^{15,16}$ of the $\mathrm{Ni}_{80} \mathrm{Fe}_{20}$ film is generated in the pentacene layer. This $J_{\mathrm{S}}$ is then absorbed into the Pd layer. The absorbed $J_{\mathrm{S}}$ is converted into a charge current as a result of the inverse spin-Hall effect (ISHE) ${ }^{20}$ in Pd and detected as an electromotive force, $E,{ }^{17-21}$ which is expressed as

$$
\vec{E} \propto \theta_{S H E} \vec{J}_{S} \times \vec{\sigma}
$$
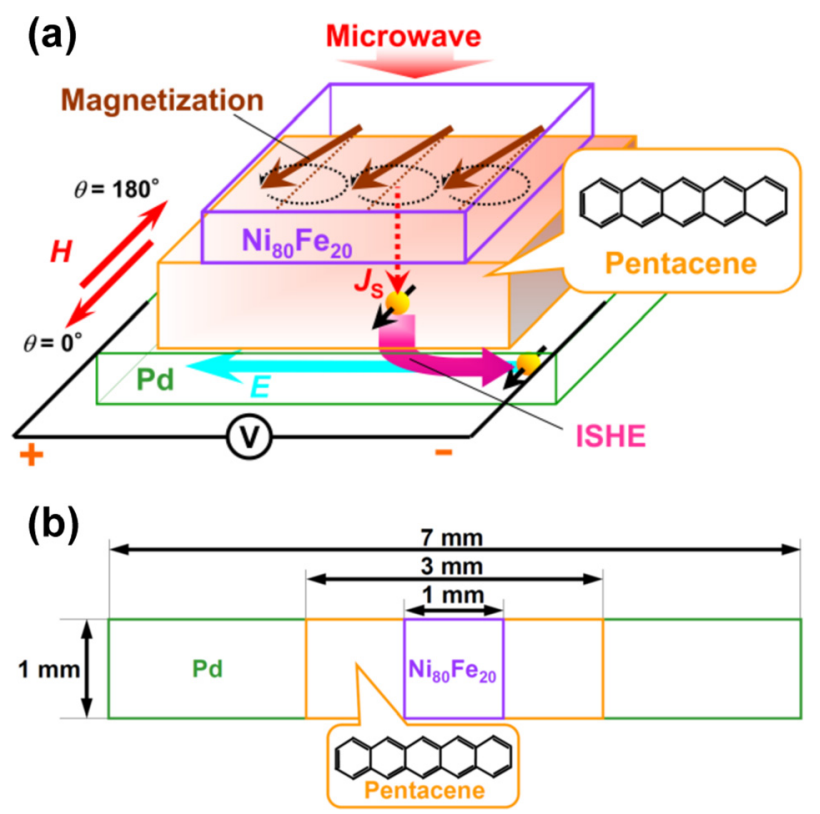

FIG. 1. (a) Bird's-eye-view and (b) top-view illustrations of our sample and orientations of external applied magnetic field $H$ used in the experiments. $J_{\mathrm{S}}$ and $E$ correspond, respectively, to the spin current generated in the pentacene film by spin-pumping and the electromotive forces due to the ISHE in Pd. 
where $\theta_{\mathrm{SHE}}$ is the spin-Hall angle, which is the efficiency of conversion from a spin current to a charge current, and $\sigma$ is the spin-polarization vector in the $J_{\mathrm{S}}$. That is, if electromotive force due to the ISHE in Pd is detected under the FMR of $\mathrm{Ni}_{80} \mathrm{Fe}_{20}$, it is clear evidence for spin transport in a pentacene film.

Electron beam (EB) deposition was used to deposit Pd (Furuuchi Chemical Co., Ltd., 99.99\% purity) to a thickness of $10 \mathrm{~nm}$ on a thermally-oxidized silicon substrate, under a vacuum pressure of $<10^{-6} \mathrm{~Pa}$. Next, also under a vacuum pressure of $<10^{-6} \mathrm{~Pa}$, pentacene molecules (Sigma Aldrich Co., Ltd.; sublimed grade, $99.9 \%$ ) were thermally evaporated through a shadow mask. The deposition rate and the substrate temperature during pentacene depositions were set to $0.1 \mathrm{~nm} / \mathrm{s}$ and RT, respectively, similar conditions under which pentacene films show high crystallinity. ${ }^{22}$ The pentacene layer thickness, $d$, was varied between 0 and $100 \mathrm{~nm}$. Finally, $\mathrm{Ni}_{80} \mathrm{Fe}_{20}$ (Kojundo Chemical Lab. Co., Ltd., 99.99\%) was deposited by EB deposition through another shadow mask, under a vacuum pressure of $<10^{-6} \mathrm{~Pa}$. During $\mathrm{Ni}_{80} \mathrm{Fe}_{20}$ deposition, the sample substrate was cooled with a cooling medium of $-2{ }^{\circ} \mathrm{C}$, to prevent the deposited molecular films from breaking. As a control experiment, samples with a $\mathrm{Cu}$ layer, instead of the Pd layer, were prepared.

We used a microwave $\mathrm{TE}_{011}$-mode cavity in an electron spin resonance (ESR) system (JEOL, JES-TE300) to excite FMR in $\mathrm{Ni}_{80} \mathrm{Fe}_{20}$, and a nano-voltmeter (Keithley Instruments, $2182 \mathrm{~A})$ to detect electromotive forces from the samples. Leading wires for detecting the output voltage properties were directly attached with silver paste at both ends of the Pd (or $\mathrm{Cu}$ ) layer. All of the measurements were performed at RT.

Figure 2(a) shows the FMR spectrum of a sample with a Pd layer and with the $d$ of $50 \mathrm{~nm}$ at an external magnetic field orientation angle $\theta$ of $0^{\circ}$, under a microwave power of $200 \mathrm{~mW}$. The FMR field $\left(H_{\mathrm{FMR}}\right)$ of the $\mathrm{Ni}_{80} \mathrm{Fe}_{20}$ film is $120 \mathrm{mT}$ and the $4 \pi M_{\mathrm{s}}$ of the $\mathrm{Ni}_{80} \mathrm{Fe}_{20}$, where $M_{\mathrm{S}}$ is the saturation magnetization of the $\mathrm{Ni}_{80} \mathrm{Fe}_{20}$ film, is estimated to be $729 \mathrm{mT}$ at a microwave frequency $f$ of $9.45 \mathrm{GHz}$ and under FMR conditions in the in-plane field

$$
\frac{\omega}{\gamma}=\sqrt{H_{F M R}\left(H_{F M R}+4 \pi M_{S}\right)},
$$

where $\omega$ and $\gamma$ are the angular frequency $2 \pi f$ and the gyromagnetic ratio of $1.86 \times 10^{11} \mathrm{~T}^{-1} \mathrm{~s}^{-1}$ of $\mathrm{Ni}_{80} \mathrm{Fe}_{20}$, respectively. ${ }^{21}$ Fig. 2(b) shows the output voltage properties of the same sample shown in Fig. 2(a); the circles represent experimental data and the solid lines are the curve fit obtained using the equation ${ }^{17-21}$

$$
\begin{aligned}
V(H)= & V_{I S H E} \frac{\Gamma^{2}}{\left(H-H_{F M R}\right)^{2}+\Gamma^{2}} \\
& +V_{A s y m} \frac{-2 \Gamma\left(H-H_{F M R}\right)}{\left(H-H_{F M R}\right)^{2}+\Gamma^{2}},
\end{aligned}
$$

where $\Gamma$ denotes the damping constant $(11.3 \mathrm{mT}$ in this study). The first and second terms in Eq. (3) correspond to the symmetry term to $H$ corresponding to the ISHE and the asymmetry term to $H$ (e.g., anomalous Hall effect ${ }^{17-21}$ and other effects showing the same asymmetric voltage behavior

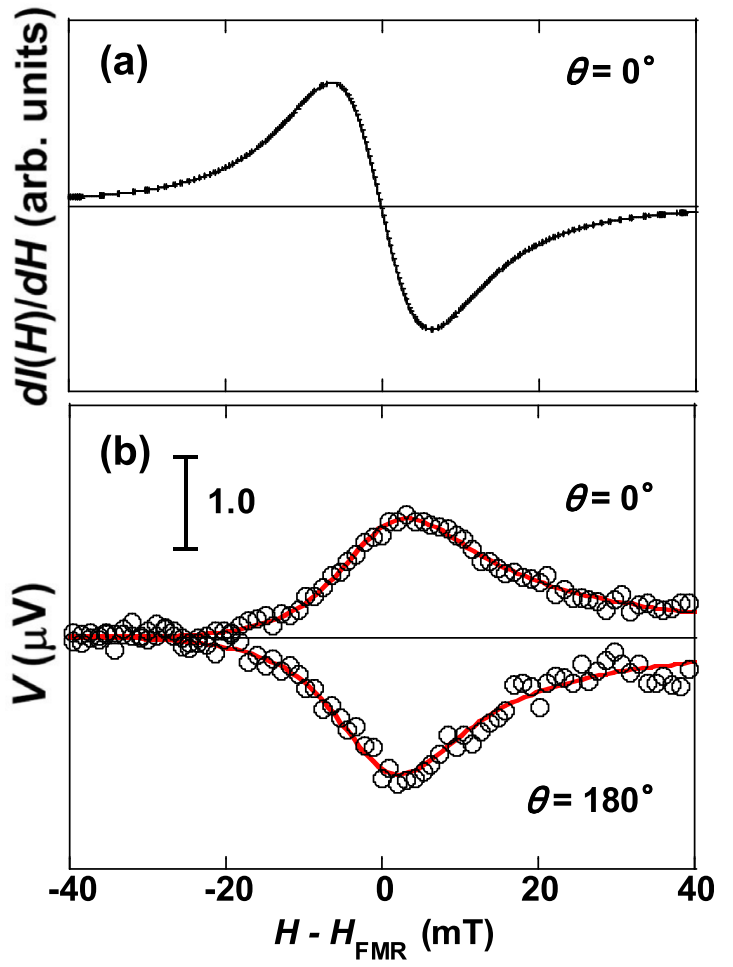

FIG. 2. (a) FMR spectrum and (b) output voltage properties of a sample with a Pd layer and with a pentacene film thickness $d$ of $50 \mathrm{~nm}$, under a microwave power of $200 \mathrm{~mW}$.

relative to the $H$, like parasitic capacitances), respectively. $V_{\text {ISHE }}$ and $V_{\text {Asym }}$ correspond to the coefficients of the first and second terms in Eq. (3). Output voltages are observed at $H_{\mathrm{FMR}}$ at $\theta$ of $0^{\circ}$ and $180^{\circ}$. Notably, the output voltage changes sign between $\theta$ values of $0^{\circ}$ and $180^{\circ}$. This sign inversion of voltage in $\mathrm{Pd}$ associated with the magnetization reversal in $\mathrm{Ni}_{80} \mathrm{Fe}_{20}$ is characteristic of ISHE. ${ }^{17,19,21}$

As a control experiment, we tested samples with a $\mathrm{Cu}$ layer, where the spin-orbit interaction is relatively weak, instead of the Pd layer. Figure 3(a) shows the FMR spectrum of a sample with a $\mathrm{Cu}$ layer and with the $d$ of $50 \mathrm{~nm}$ at the $\theta$ of $0^{\circ}$, under a microwave power of $200 \mathrm{~mW}$. Fig. 3(b) shows output voltage properties for the same sample as in Fig. 3(a), where no clear electromotive force was observed at $\theta$ values of $0^{\circ}$ and $180^{\circ}$. As another control experiment, we studied the microwave power $(P)$ dependence of the electromotive forces; the results are shown in Fig. 4. The $V_{\text {ISHE }}$ increases in proportion to the increase in $P$. The above results suggest that the electromotive forces at the FMR field $\left(H-H_{\mathrm{FMR}}=0\right)$ observed for the sample with a Pd layer (see Fig. 2(b)) are mainly due to the ISHE in Pd, that is, spinpump-induced spin transport is achieved in an evaporated pentacene film at RT.

Figure 5 shows the $d$ dependence of (a) $4 \pi M_{\mathrm{s}}$ calculated via Eq. (2) and of (b) $V_{\text {ISHE }}$ estimated via Eq. (3). With increasing $d, M_{\mathrm{s}}$ decreases slightly, while $V_{\mathrm{ISHE}}$ clearly decreases. The data for the pentacene-free case $(d=0 \mathrm{~nm})$ are plotted as open circles in Fig. 5(b). $V_{\text {ISHE }}$ in the pentacene-free case, however, includes extrinsic effects, e.g., the electromotive force due to $\mathrm{Ni}_{80} \mathrm{Fe}_{20}$ itself. ${ }^{23}$ Thus, using all data except those for $d=0 \mathrm{~nm}$, we estimated the spin diffusion length of the evaporated-pentacene film $\lambda_{\mathrm{s}}$ to be 

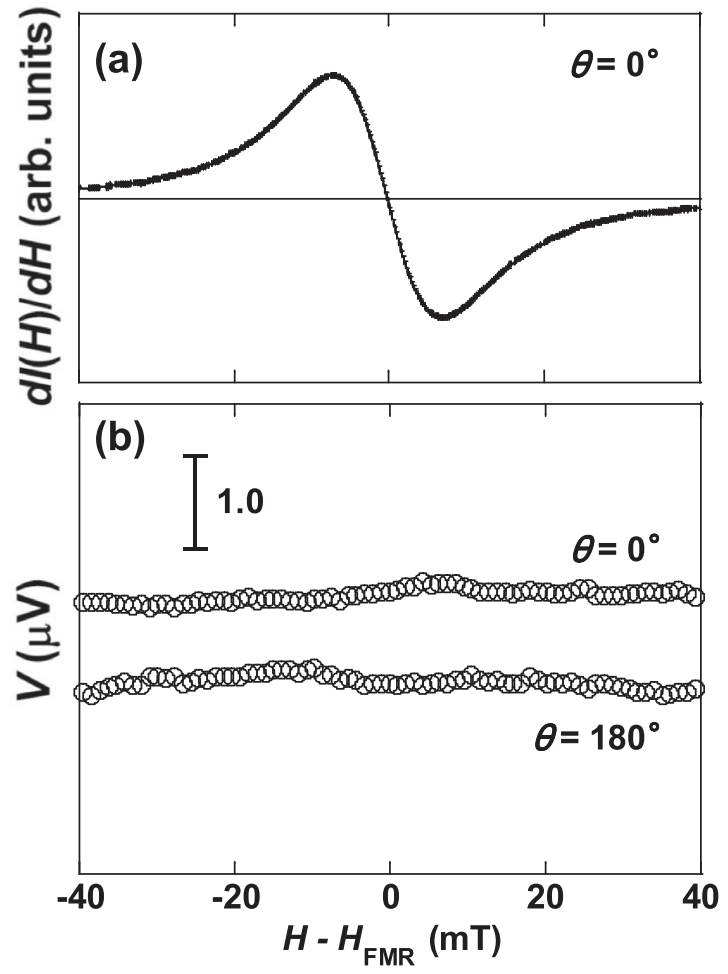

FIG. 3. (a) FMR spectrum and (b) output voltage properties of a sample with a $\mathrm{Cu}$ layer and with a pentacene film thickness $d$ of $50 \mathrm{~nm}$, under a microwave power of $200 \mathrm{~mW}$.

$42 \pm 10 \mathrm{~nm}$ at RT under the assumption ${ }^{4,17}$ of an exponential decay of the spin current in the pentacene film. The dashed lines in Fig. 5 represent the results of this estimation. To validate these estimates, we also estimated $\lambda_{\mathrm{s}}$ in another way, ${ }^{17}$ using the linewidth of the FMR spectra, the $4 \pi M_{\mathrm{s}}$ calculated via Eq. (2), and the experimentally obtained electromotive forces results. The detailed calculation method is described in Ref. 17. The real part of the mixing conductance at the interface between $\mathrm{Ni}_{80} \mathrm{Fe}_{20}$ and the Pd layer, $g_{r}^{\uparrow \downarrow}$, which could be thought of as the transmittance of the spin current, and the generated spin current density in the Pd layer, $j_{\mathrm{s}}{ }^{\prime}$, are

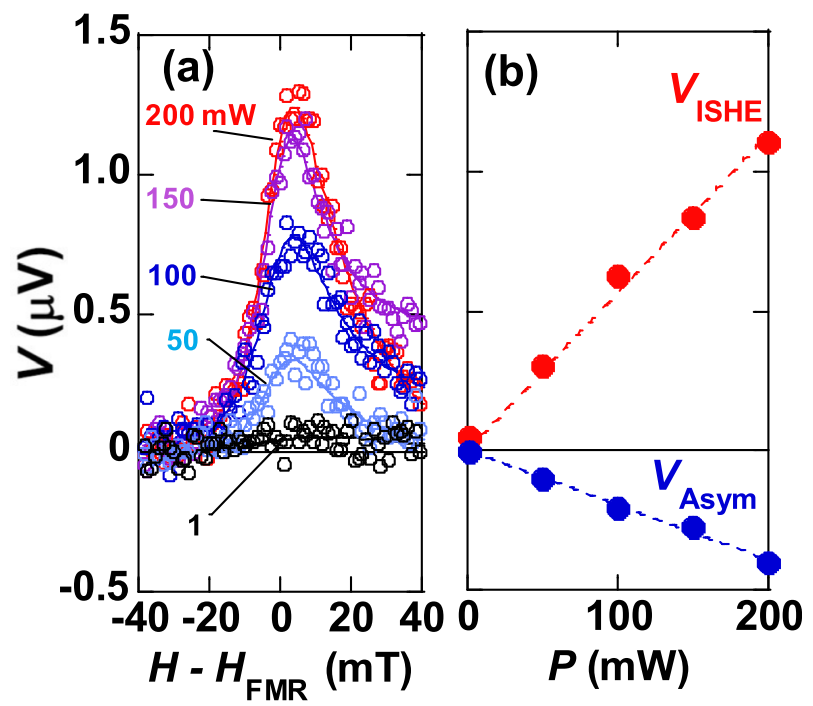

FIG. 4. (a) Microwave power $(P)$ dependence of electromotive force and (b) analysis results obtained with Eq. (2). $V_{\text {ISHE }}$ and $V_{\text {Asym }}$ correspond to the coefficients of the first and second terms in Eq. (2).

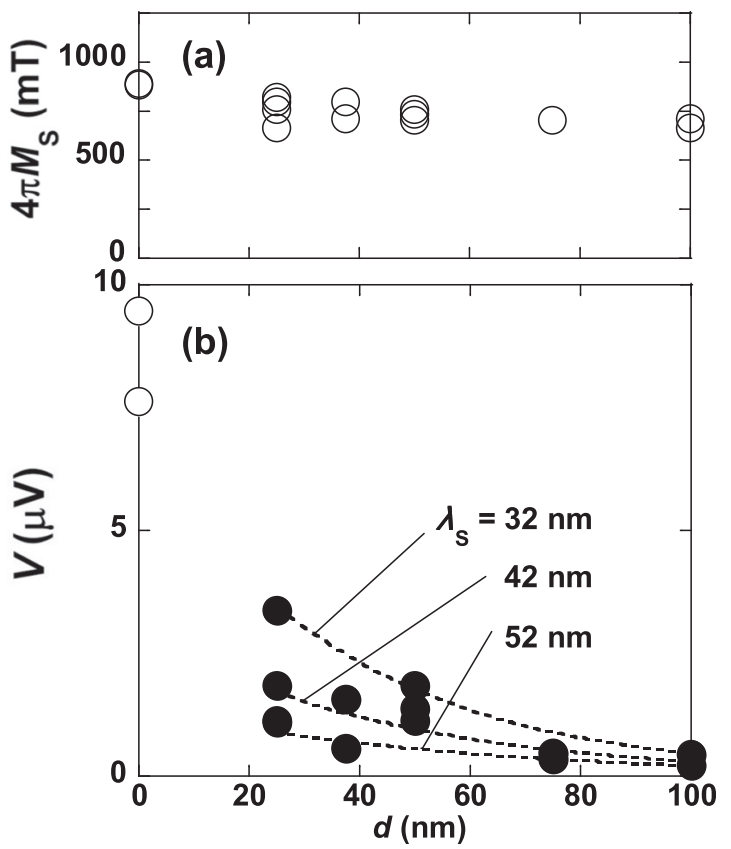

FIG. 5. Dependence of (a) $4 \pi M_{\mathrm{s}}$ ( $M_{\mathrm{s}}$ : saturation magnetization), calculated by Eq. (1), and of (b) $V_{\text {ISHE }}$ estimated by Eq. (2), on the pentacene film thickness $(d)$. Open and closed circles in (b) are the experimental data. The dashed lines in (b) are curve fits under the assumption of exponential decay.

calculated to be $2.52 \times 10^{19} \mathrm{~m}^{-2}$ and $7.31 \times 10^{-10} \mathrm{~J} \mathrm{~m}^{-2}$, respectively. This $g_{r}^{\uparrow \downarrow}$ is almost the same as that obtained in experiments involving spin-pump-induced spin transport in $p$-type $\mathrm{Si}^{17}$ Meanwhile, the $j_{\mathrm{s}}{ }^{\prime}$ in the present study is by two orders of magnitude smaller than in the case of the $p$-Si. ${ }^{17}$ Using the spin diffusion length of $\mathrm{Pd}, 9 \mathrm{~nm}^{24}$ and the $\theta_{\text {SHE }}$ of $\mathrm{Pd}, 0.01,{ }^{21}$ the $\lambda_{\mathrm{s}}$ is estimated to be about $38.5 \mathrm{~nm}$. The above two $\lambda_{\mathrm{s}}$ estimates are almost the same, although the $\lambda_{\mathrm{s}}$ value of $30-40 \mathrm{~nm}$ at RT is comparable to or shorter than the spin diffusion lengths of other molecules: $\sim 150 \mathrm{~nm}$ for a $\pi$-conjugated polymer (PBTTT) ${ }^{4} \sim 150 \mathrm{~nm}$ for a $p$-type conducting polymer (PEDOT:PSS), ${ }^{10}$ and $\sim 50 \mathrm{~nm}$ for a lowmolecular-weight $n$-type molecule $\left(\mathrm{Alq}_{3}\right){ }^{8,9}$ Pentacene is a low-molecular-weight $p$-type semiconductor. Thus, the polarity of the major carrier of the molecules is not strongly affected by the shortening of the spin diffusion length. If a detailed investigation of the relationship between the carrier polarity and the spin transport mechanism is necessary, a study of spin transport using ambipolar molecules would be effective. Polymers, such as PBTTT and PEDOT:PSS, tend to possess longer spin diffusion lengths than low-molecularweight molecules, such as $\mathrm{Alq}_{3}$ and pentacene. We checked crystallinity of our pentacene film grown on the Pd layer by using an X-ray diffractometer (See supplementary mate$\mathrm{rial}^{25}$ ). In our pentacene films, the pentacene molecules might partially be oriented. In general, $\mathrm{Alq}_{3}$ molecular film has amorphous state. Comparing pentacene with $\mathrm{Alq}_{3}$, the crystallinity of molecular films may be related to the spin diffusion length. That is, the higher the crystallinity of a molecular film is, the longer the spin diffusion length of the molecular film might be. Thus, to improve the crystallinity of pentacene films is an indispensable issue to clarify the mechanism of spin transport in a pentacene film. The mechanism of spin transport among the above molecules may be 
different because the charge transport mechanism is different for each. At present, the reason why polymers show longer spin diffusion lengths is still unclear, except for the crystallinity of molecular films. More studies of the spin transport in various molecules having various charge transport mechanisms and having high crystallinity are eagerly awaited to clarify the spin-transport mechanism in molecular films.

In summary, spin transport properties of evaporatedpentacene films were studied at RT by using spin-pumping for spin injection and using the ISHE in non-magnetic metals in the spin detection methods. We achieved spin transport in evaporated-pentacene films; the spin diffusion length in pentacene was estimated to be above $30 \mathrm{~nm}$ at RT. This is clear evidence for pure spin current in molecular films prepared by thermal evaporation, which paves the way to moleculebased spintronic devices.

The authors thank to Mr. Yutaka Ohkawahara and Professor Dr. Masaaki Nakayama in Osaka City Univ. Japan, for helping to evaluate the crystallinity of pentacene films. This research was partly supported by a Grant-in-Aid from the Japan Society for the Promotion of Science (JSPS) for Scientific Research (B) (26286039 (to E.S.)), a Grant-in-Aid from the Japan Society for the Promotion of Science (JSPS) for Scientific Research (B) (24350076 (to Y.Te.)), and Jyuten Kenkyu (B) from Osaka City University.

${ }^{1}$ M. Shiraishi and T. Ikoma, Physica E 43, 1295 (2011).

${ }^{2}$ M. Ohishi, M. Shiraishi, R. Nouchi, T. Nozaki, T. Shinjo, and Y. Suzuki, Jpn. J. Appl. Phys., Part 2 46, L605 (2007).

${ }^{3}$ R. Ohshima, A. Sakai, Y. Ando, T. Shinjo, K. Kawahara, H. Ago, and M. Shiraishi, Appl. Phys. Lett. 105, 162410 (2014).

${ }^{4}$ S. Watanabe, K. Ando, K. Kang, S. Mooser, Y. Vaynzof, H. Kurebayashi, E. Saitoh, and H. Sirringhaus, Nat. Phys. 10, 308 (2014).
${ }^{5}$ T. Shimada, H. Nogawa, T. Noguchi, Y. Furubayashi, Y. Yamamoto, Y. Hirose, T. Hitosugi, and T. Hasegawa, Jpn. J. Appl. Phys., Part 1 47, 1184 (2008).

${ }^{6}$ T. Ikegami, I. Kawayama, M. Tonouchi, S. Nakao, Y. Yamashita, and H. Tada, Appl. Phys. Lett. 92, 153304 (2008).

${ }^{7}$ X. Sun, M. Gobbi, A. Bedoya-Pinto, O. Txoperena, F. Golmar, R. Llopis, A. Chuvilin, F. Casanova, and L. Hueso, Nat. Commun. 4, 2794 (2013).

${ }^{8}$ Z. H. Xiong, D. Wu, Z. V. Vardeny, and J. Shi, Nature 427, 821 (2004).

${ }^{9}$ E. Shikoh, A. Fujiwara, Y. Ando, and T. Miyazaki, Jpn. J. Appl. Phys., Part 1 45, 6897 (2006).

${ }^{10}$ M. Kimata, D. Nozaki, Y. Niimi, H. Tajima, and Y. Otani, Phys. Rev. B 91, 224422 (2015).

${ }^{11}$ H. Cheng, Y. Mai, W. Chou, and L. Chang, Appl. Phys. Lett. 90, 171926 (2007).

${ }^{12}$ O. Ostroverkhova, D. G. Cooke, S. Shcherbyna, R. F. Egerton, F. A. Hegmann, R. R. Tykwinski, and J. E. Anthony, Phys. Rev. B 71, 035204 (2005).

${ }^{13}$ G. Schmidt, D. Ferrand, L. W. Molenkamp, A. T. Filip, and B. J. van Wees, Phys. Rev. B 62, R4790 (2000).

${ }^{14}$ A. Fert and H. Jaffres, Phys. Rev. B 64, 184420 (2001).

${ }^{15}$ S. Mizukami, Y. Ando, and T. Miyazaki, Phys. Rev. B 66, 104413 (2002).

${ }^{16}$ Y. Tserkovnyak, A. Brataas, and G. E. W. Bauer, Phys. Rev. Lett. 88, 117601 (2002).

${ }^{17}$ E. Shikoh, K. Ando, K. Kubo, E. Saitoh, T. Shinjo, and M. Shiraishi, Phys. Rev. Lett. 110, 127201 (2013).

${ }^{18}$ A. Yamamoto, Y. Ando, T. Shinjo, T. Uemura, and M. Shiraishi, Phys. Rev. B 91, 024417 (2015).

${ }^{19}$ S. Dushenko, M. Koike, Y. Ando, T. Shinjo, M. Myronov, and M. Shiraishi, Phys. Rev. Lett. 114, 196602 (2015).

${ }^{20}$ E. Saitoh, M. Ueda, H. Miyajima, and G. Tatara, Appl. Phys. Lett. 88, 182509 (2006).

${ }^{21}$ K. Ando and E. Saitoh, J. Appl. Phys. 108, 113925 (2010).

${ }^{22}$ C. D. Dimitrakopoulos and P. R. L. Malenfant, Adv. Mater. 14, 99 (2002).

${ }^{23}$ A. Tsukahara, Y. Ando, Y. Kitamura, H. Emoto, E. Shikoh, M. P. Delmo, T. Shinjo, and M. Shiraishi, Phys. Rev. B 89, 235317 (2014).

${ }^{24}$ J. Foros, G. Woltersdorf, B. Heinricha, and A. Brataas, J. Appl. Phys. 97, 10A714 (2005).

${ }^{25}$ See supplementary material at http://dx.doi.org/10.1063/1.4938132 for XRD measurement of a pentacene film grown on a Pd layer. 